\title{
ON THE BLOCK STRUCTURE OF PBIB DESIGNS
}

\author{
M.L. CHANDAK \\ Department of Mathematics and Statistics \\ J.N. Agricultural University \\ Jabalpur-4 \\ M.P. , India
}

(Received August 11, 1978 and in revised form February 2, 1979)

ABSTRACT. Let $\left\{\theta, \theta_{1}, \ldots, \theta_{n_{1}}\right\}$ denote the set having any treatment $\theta$ and its $n_{1}$ first associates in a PBIB design with $m(\geq 2)$ associate classes. A theorem on distribution of the elements of this set among the blocks of the block design is proved and results related to certain well known association schemes of three associate classes are obtained.

KEY WORDS AND PHRASES. PBIB Designs, Rectangular Association Scheme, Cubic Association Scheme.

1980 MATHEMATICS SUBJECT CLASSIFICATION CODES. Primary 62K05, 62K10, 62K15; Secondary 05B05, 05BTO.

1. INTRODUCTION.

Bose and Connor [1] have shown that for a semi-regular group divisible design with two associate classes the treatments in any group are equally distributed among the blocks of the design. This result was later generalized by Murty [2] for any PBIB design with two associate classes. The purpose 
of this note is to generalize his theorem for PBIB designs with $m(\geq 2)$ associate classes. Some connected results for three associate PBIB designs are then obtained in section 5 .

\section{ASSOCIATION SCHEMES AND PBIB DESIGNS.}

Given $v$ treatments, $1,2, \ldots, v$ a relation satisfying the following conditions is said to be an association scheme with $m$ classes:

(a) Any two treatments are either 1st, 2nd,..., or $m$ th associates, the relation of association being symmetric, i.e. if treatment $\alpha$ is the $i$ th associate of treatment $\beta$, then $\beta$ is the $i$ th associate of treatment $\alpha$.

(b) Each treatment has $n_{1}$, $i$ th associates, the number $n_{i}$ being independent of the treatment taken.

(c) If any two treatments $\alpha$ and $\beta$ are $i$ th associates then the number of treatments which are $j$ th associates of $\alpha$ and $k$ th associates of $\beta$ is $p_{j k}^{i}$ and is independent of the pair of $i$ th associates $\alpha$ and $\beta$.

The numbers $v, n_{i}, p_{j k}^{i}, 1 \leq i, j, k \leq m$, are the parameters of the association scheme. If we have an association scheme with $m$ - classes, then we get a PBIB design with $r$ replications, $b$ blocks, and block size $k$ based on the association scheme provided we can arrange the $v$ treatments into b blokks such that
(i) each block contains $\mathrm{k}$ distinct treatments
(1i) each treatment is contained in $r$ blocks
(iii) if two treatments $\alpha$ and $\beta$ are $i$ th associates, then they

occur together in $\lambda_{i}$ blocks (not all $\lambda_{i}$ 's equal), the number $\lambda_{i}$ being independent of the particular pair of $i$ th associates $\alpha$ and $\beta(1 \leq i \leq m)$.

\section{SOME KNOWN ASSOCIATION SCHEMES WITH THREE ASSOCIATE CLASSES}

(a) Cubic association scheme. (cf. Raghavarao et al [3] ). Let there be $v=s^{3}$ treatments denoted by $(\alpha, \beta, \gamma) \cdot(\alpha, \beta, \gamma,=1$, $2, \ldots, s)$. We define the distance $\delta$ between two treatments $(\alpha, \beta, \gamma)$ and 
$\left(\alpha^{\prime}, \beta^{\prime}, \gamma^{\prime}\right)$ to be the number of non-null elements in $\left(\alpha-\alpha^{\prime}, \beta-\beta^{\prime}, \gamma-\gamma^{\prime}\right)$. Any two treatments are 1 st, 2 nd or 3 rd associates according as $=1,2$, or 3 respectively.

(b) Rectangular association scheme. (Vartak, [6], Shah [5]).

If the treatments $v=v_{1} \cdot v_{2}$ can be arranged in the form of a rectangle of $v_{1}$ rows and $v_{2}$ colums, then the first associates of any treatment are the other $\left(v_{2}-1\right)$ treatments of the same row, the second associates are the other $\left(v_{1}-1\right)$ treatments of the same column and the remaining $\left(v_{1}-1\right)$. $\left(v_{2}-1\right)$ treatments are the third associates.

The reader may consult the references for examples of schemes and their parameters.

4. DISTRIBUTION OF THE $\left(n_{1}+1\right)$ TREATMENTS (VIZ. ANY TREATMENT $\theta$ AND ITS $n_{1}$ FIRST ASSOCIATES) AMONG THE b BLOCKS OF THE DESIGN

Let $\theta$ be any of the $v$ treatments and let $\theta_{1}, \theta_{2}, \ldots, \theta_{n_{1}}$ be the $n_{1}$ first associates of $\theta$. From among these $\left(n_{1}+1\right)$ treatments let $e_{j}$ treatments occur in the $j$ th block $(j=12, \ldots, b)$. We now prove the following:

THEOREM. If in any PBIB design with $\mathrm{m}(\geq 2)$ associate classes

$\sum_{t=1}^{m} p_{1 t}^{1} \lambda_{t}=\frac{\left(n_{1}+1\right) r}{n_{1}}\left\{\frac{\left(n_{1}+1\right) k}{v}-1\right\}-2 \lambda_{1}$

then $\left(n_{1}+1\right) k$ is divisible by $v$. Further every block of the design contains $\left(n_{1}+1\right) k / v$ treatments among the $\left(n_{1}+1\right)$ treatments $\theta, \theta_{1}, \theta_{2}, \ldots, \theta_{1}$

PROOF. We have

$\sum_{j=1}^{b} e_{j}=\left(n_{1}+1\right) r$.

$\sum_{j=1}^{b} e_{j}\left(e_{j}-1\right)=n_{1}\left\{2 \lambda_{1}+\sum_{t=1}^{m} p_{1 t}^{1} \lambda_{t}\right\}$, 
Defining $\bar{e}=b^{-1} \sum_{j=1}^{b} e_{j}=\left(n_{1}+1\right) k / v$.

Now we have

$\sum_{j=1}^{b}\left(e_{j}-\bar{e}\right)^{2}=\sum_{j=1}^{b} e_{j}\left(e_{j}-1\right)+\sum_{j=1}^{b} e_{j}-b \bar{e}^{2}$

$=n_{1}\left\{2 \lambda_{1}+\sum_{t=1}^{m} p_{1 t}^{1} \lambda_{t}\right\}+\left(n_{1}+1\right) r-\frac{b\left(n_{1}+1\right)^{2} k^{2}}{v}=0 . \quad$ from (4.1)

Therefore $e_{1}=e_{2}=\ldots=e_{b}=\bar{e}=\frac{\left(n_{1}+1\right) k}{v}$. Since $e_{j}(j=1,2, \ldots, b)$ must be integral $v$ divides $\left(n_{1}+1\right) k$.

This completes the proof of the theorem.

5. APPLICATIONS IN PBIB DESIGNS WITH THREE ASSOCIATE CLASSES.

Let $N=\left(n_{1 j}\right)$ be the $v \times$ b incidence matrix of the design, where $n_{1 j}=1$ or 0 according as the $i$ th treatment occurs in the $j$ th block or not.

(a) Designs based on cubic association scheme.

Raghayarao et al (1964) obtained the characteristic roots of NN' of such designs, Let $P_{0}, P_{1}, P_{2}$, and $P_{3}$ denote these roots, then we have

$$
\begin{aligned}
& P_{0}=r+3(s-1) \lambda_{1}+3(s-1)^{2} \lambda_{2}+(s-1)^{3} \lambda_{3}=r k \\
& P_{1}=r+(2 s-3) \lambda_{1}+(s-1)(s-3) \lambda_{2}-(s-1)^{2} \lambda_{3}, \\
& P_{2}=r+(s-3) \lambda_{1}-(2 s-3) \lambda_{2}+(s-1) \lambda_{3}, \\
& P_{3}=r-3 \lambda_{1}+3 \lambda_{2}-\lambda_{3} .
\end{aligned}
$$

We prove the following:

THEOREM 5.1. If in any PBIB design based on cubic association scheme

$$
\frac{v}{k}-I=\frac{3 s(s-1)}{(3 s-2)^{2} P_{0}}\left\{(5 s-4) P_{1}+8(s-1)^{2} P_{2}+(s-1)^{2}(3 s-4) P_{3}\right\}
$$


then $(3 s-2) k$ is divisible by $s^{3}$. Further every block of the design contains $(3 \mathrm{~s}-2) \mathrm{k} / \mathrm{s}^{3}$ treatments from among the $\left(\mathrm{n}_{1}+1\right)$ treatments $\theta, \theta_{1}, \theta_{2}, \ldots, \theta_{n_{1}}$.

PROOF. Condition $(4.1)$ reduces to

$\sum_{t=1}^{3} p_{1 t}^{1} \lambda_{t}=\frac{r\left(n_{1}+1\right)}{n_{1}}\left\{\frac{\left(n_{1}+1\right) k}{v}-1\right\}-2 \lambda_{1}$.

In cubic association scheme one can easily see

$n_{1}=3(s-1), p_{11}^{1}=s-2, p_{12}^{1}=2(s-1), p_{13}^{1}=0$.

So, we have

$(s-2) \lambda_{1}+2(s-1) \lambda_{2}=\frac{r(3 s-2)}{3(s-1)}\left\{\frac{(3 s-2) k}{v}-1\right\}-2 \lambda_{1}$.

Substituting the values of $\lambda_{1}$ and $\lambda_{2}$ from (5.1) and thereafter some simplifications lead to (5.2). An appeal to the Theorem of section 4 completes the proof.

EXAMPLE: consider $\mathrm{v}=2^{3}=8$, then the treatments are (111) (112) (121) (122) (211) (212) (221) (222). The scheme parameters will be $\mathrm{v}=8, \mathrm{n}_{1}=3, \mathrm{n}_{2}=3, \mathrm{n}_{3}=1 ; \quad \mathrm{p}_{j \mathrm{k}}^{1}=\left[\begin{array}{lll}0 & 2 & 0 \\ 2 & 0 & 1 \\ 0 & 1 & 0\end{array}\right], \mathrm{p}_{j \mathrm{k}}^{2}=\left[\begin{array}{lll}2 & 0 & 1 \\ 0 & 2 & 0 \\ 1 & 0 & 0\end{array}\right], \mathrm{p}_{j k}^{3}=\left[\begin{array}{lll}0 & 3 & 0 \\ 3 & 0 & 0 \\ 0 & 0 & 0\end{array}\right]$.

The design with parameters

$\mathrm{b}=6, \mathrm{r}=3, \mathrm{k}=4, \lambda_{1}=1, \lambda_{2}=1, \lambda_{3}=3$

satisfies the requirements of the theorem and therefore allows even distribution

of a given treatment and its first associates among the blocks, as can be seen from the following;

$\mathrm{B}_{1}:(111,112,222,221) ; \mathrm{B}_{4}:(112,122,221,211)$;

$\mathrm{B}_{2}:(111,121,222,212) ; \mathrm{B}_{5}:(112,212,221,121)$;

$B_{3}:(111,211,222,122) ; B_{6}:(121,122,211,212)$; 
(b) Designs based on rectangular association scheme.

Vartak (1959) as pointed out in Saha (1966) proved that the

characteristic roots of $\mathrm{NN}^{\prime}$ of this design are $\mathrm{P}_{0}=\mathrm{rk}, \mathrm{P}_{1}=\mathrm{r}-\lambda_{1}+\left(\mathrm{v}_{1}-1\right)$. $\left(\lambda_{2}-\lambda_{3}\right), \quad P_{2}=r-\lambda_{2}+\left(v_{2}-1\right)\left(\lambda_{1}-\lambda_{3}\right), \quad P_{3}=r-\lambda_{1}-\lambda_{2}+\lambda_{3}$.

THEOREM 5.2. If in a PBIB design based on rectangular association scheme.

$\frac{v}{k}-1=\left(\frac{v_{2}-1}{P_{0}}\right)\left\{P_{1}+\left(v_{1}-1\right) P_{3}\right\}$,

then $k$ is divisible by $v_{1}$. Further every block of the design contains $k / v_{1}$ treatments among the $\left(n_{1}+1\right)$ treatments $\theta, \theta_{1}, \ldots, \theta_{n_{1}}$.

PROOF. Here we have $n_{1}=v_{2}-1, p_{11}^{1}=v_{2}-2, p_{12}^{1}=p_{13}^{1}=0$. The Theorem can be proved similar to previous theorem.

EXAMPLE 1. Let $\mathrm{v}=2 \times 5=10$, then $\mathrm{n}_{1}=4, \mathrm{n}_{2}=1, \mathrm{n}_{3}=4$ and $\mathrm{p}_{\mathrm{jk}}^{1}=\left[\begin{array}{lll}3 & 0 & 0 \\ 0 & 0 & 1 \\ 0 & 1 & 3\end{array}\right], \quad \mathrm{p}_{\mathrm{jk}}^{2}=\left[\begin{array}{lll}0 & 0 & 4 \\ 0 & 0 & 0 \\ 4 & 0 & 0\end{array}\right], \quad \mathrm{p}_{\mathrm{jk}}^{3}=\left[\begin{array}{lll}0 & 1 & 3 \\ 1 & 0 & 0 \\ 3 & 0 & 0\end{array}\right]$

are the scheme parameters.

The design with parameters

$\mathrm{b}=10, \mathrm{r}=\mathrm{k}=4, \lambda_{1}=1, \lambda_{2}=4, \lambda_{3}=1$

satisfies the requirements of the theorem and therefore the set consisting of a given treatment and its first associates is evenly distributed among the blocks, which can be seen:

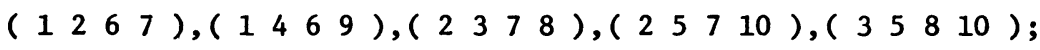

$\left(\begin{array}{llll}1 & 3 & 6 & 8\end{array}\right),\left(\begin{array}{llll}1 & 5 & 6 & 10\end{array}\right),\left(\left(\begin{array}{llll}2 & 4 & 7 & 9\end{array}\right),\left(\begin{array}{llll}3 & 4 & 8 & 9\end{array}\right),\left(\begin{array}{llll}4 & 5 & 9 & 10\end{array}\right)\right.$.

EXAMPLE 2. Let $v=3 \times 4=12$, then $n_{1}=3, n_{2}=2, n_{3}=6$ and 
$\mathrm{p}_{j k}^{1}=\left[\begin{array}{lll}2 & 0 & 0 \\ 0 & 0 & 2 \\ 0 & 2 & 4\end{array}\right], \quad \mathrm{p}_{\mathrm{jk}}^{2}=\left[\begin{array}{lll}0 & 0 & 3 \\ 0 & 1 & 0 \\ 3 & 0 & 3\end{array}\right], \quad \mathrm{p}_{\mathrm{jk}}^{3}=\left[\begin{array}{lll}0 & 1 & 2 \\ 1 & 0 & 1 \\ 2 & 1 & 2\end{array}\right]$

are the scheme parameters.

Any treatment with its first associates are evenly distributed among the blocks of the design with parameters $b=12, \mathbf{r}=\mathrm{k}=3, \lambda_{1}=0=\lambda_{2}, \lambda_{3}=1$ and the blocks are :

$\left(\begin{array}{lll}1 & 6 & 11\end{array}\right),\left(\begin{array}{lll}2 & 5 & 12\end{array}\right),\left(\begin{array}{lll}3 & 5 & 10\end{array}\right),\left(\begin{array}{lll}4 & 5 & 11\end{array}\right)$;

$\left(\begin{array}{lll}1 & 7 & 12\end{array}\right),\left(\begin{array}{lll}2 & 7 & 9\end{array}\right),\left(\begin{array}{lll}3 & 6 & 12\end{array}\right),\left(\begin{array}{lll}4 & 6 & 9\end{array}\right)$;

$\left(\begin{array}{lll}1 & 8 & 10\end{array}\right),\left(\begin{array}{lll}2 & 8 & 11\end{array}\right),\left(\begin{array}{lll}3 & 8 & 9\end{array}\right),\left(\begin{array}{lll}4 & 7 & 10\end{array}\right)$.

ACKNOWLEDGMENT. The author is grateful to the referee for his valuable suggestions for the improvement of the earlier draft of this paper.

\section{REFERENCES}

1. Bose, R. C. and Connor, W. S. Combinatorial properties of group divisible designs, Ann. Math. Stat. 23(1952) 367-383.

2. Murty, V. N. On the block structure of PBIB designs with two associate classes, Sankhyā 26(1964) 381-382.

3. Raghavarao, D. and Chandrasekhar Rao. Cubic designs, Ann. Math. Stat. 35 (1964) 389-397.

4. Shah, S. M. Bounds for the number of identical blocks in incomplete block designs. Sankhya 38 (1976) 80-86.

5. Shah, S. M. On the block structure of certain partially balanced incomplete block designs, Ann. Math. Stat. 37(1966) 1016-1020.

6. Vartak, M. N. The non-existence of certain PBIB designs, Ann. Math. Stat. $\underline{30}$ (1959) 1051-1062. 


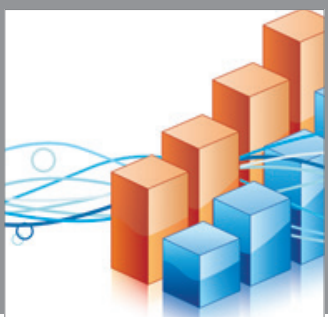

Advances in

Operations Research

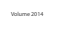

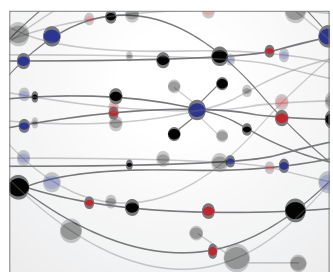

\section{The Scientific} World Journal
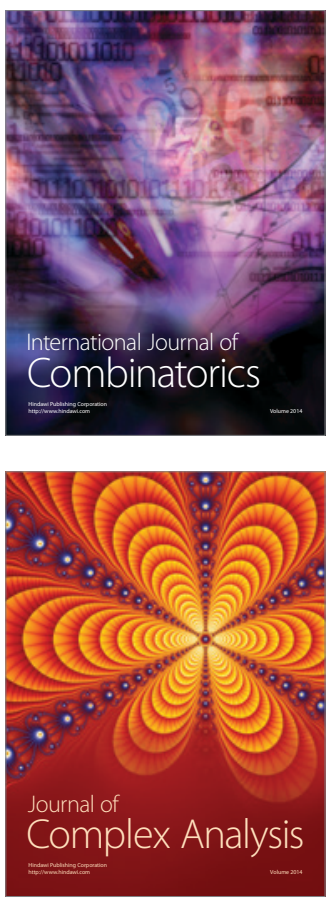

International Journal of

Mathematics and

Mathematical

Sciences
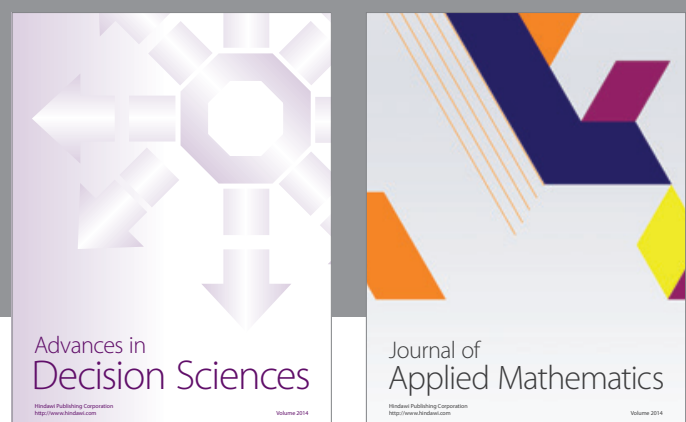

Journal of

Applied Mathematics
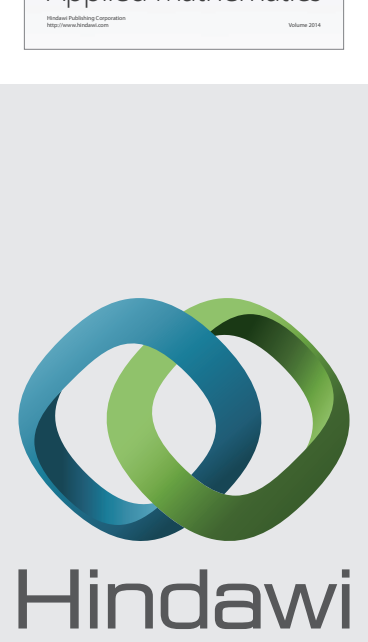

Submit your manuscripts at http://www.hindawi.com
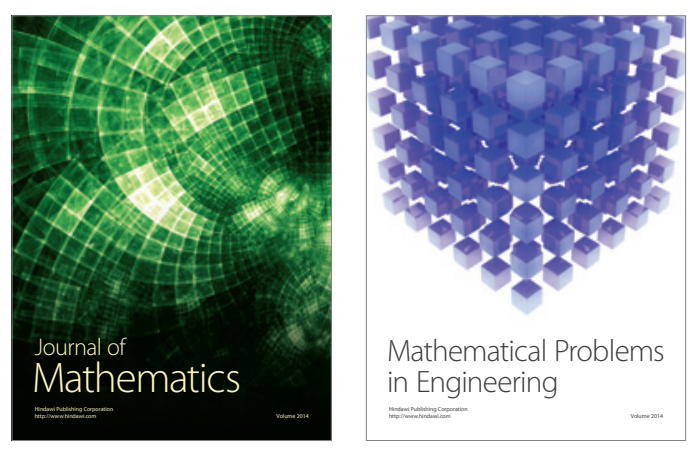

Mathematical Problems in Engineering
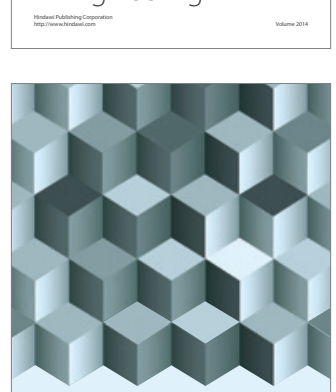

Journal of

Function Spaces
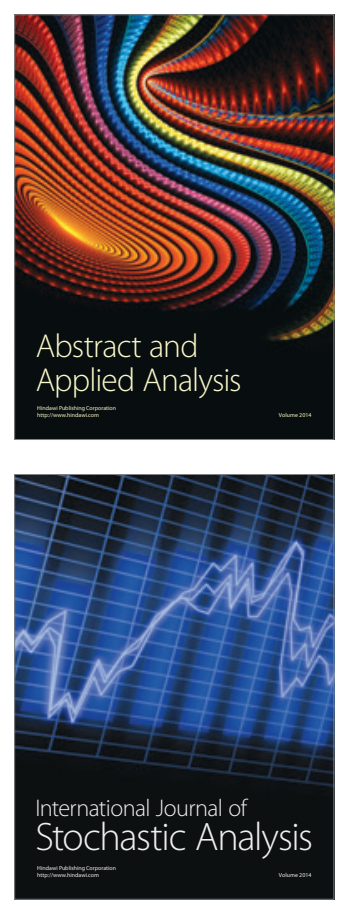

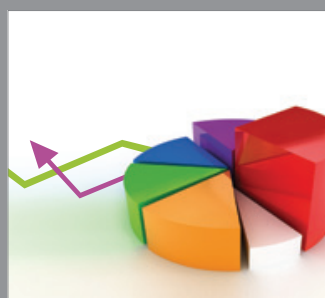

ournal of

Probability and Statistics

Promensencen
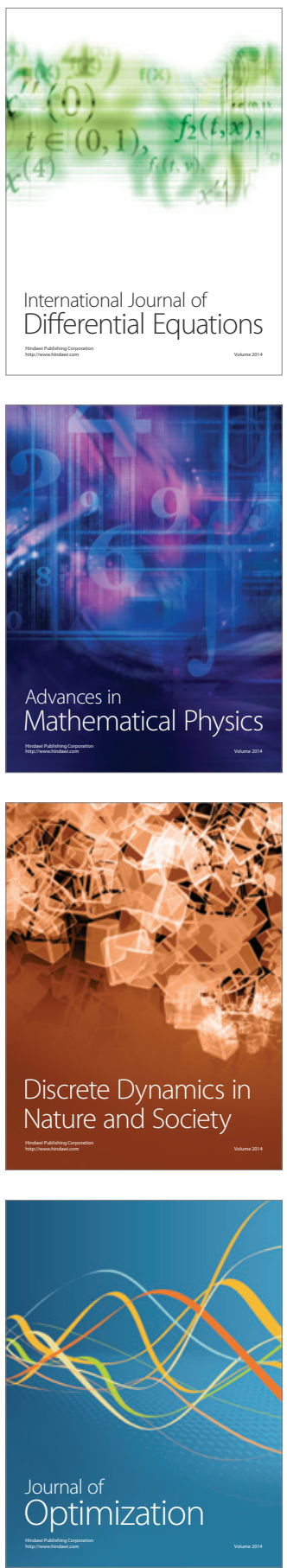